DETC2000/DAC-14232

\title{
CLOSED-FORM SWEPT VOLUME OF IMPLICIT SURFACES
}

\author{
Karim Abdel-Malek \\ Department of Mechanical Engineering and \\ Center for Computer Aided Design \\ The University of lowa \\ lowa City, IA 52246 \\ Tel. (319) 335-5676 \\ amalek@engineering.uiowa.edu
}

\author{
Jingzhou Yang \\ Department of Mechanical Engineering and \\ Center for Computer Aided Design \\ The University of lowa \\ lowa City, IA 52246
}

\author{
Denis Blackmore \\ Department of Mathematical Sciences \\ New Jersey Institute of Technology \\ Newark, NJ 07102-1982
}

\begin{abstract}
Recent developments in formulations for generating swept volumes have made a significant impact on the efficiency of employing such algorithms and on the extent to which formulations can be used in representing complex shapes. In this paper, we outline a method for employing the representation of implicit surfaces using the Jacobian rank deficiency condition presented earlier for the sweep of parametric surfaces. A numerical and broadly applicable analytic formulation is developed that yields the exact swept volume.
\end{abstract}

\section{INTRODUCTION}

Swept volumes have become increasingly important in modern Computer-Aided Design because of the need to represent the trace of objects that have experienced motion. Such applications are numerous and encompass solid modeling, manufacturing automation, robot analysis, collision detection, and computer graphics. In this report we present new results pertaining to the sweep of implicit surfaces using the Jacobian rank deficiency conditions [1-4]. Recent work published in the special edition of Computer-Aided Design [5] dedicated to the subject of swept volumes has outlined a method for trimming swept volumes.

For a review of prior work in this field, the reader is referred to Abdel-Malek and Yeh [1] for a comprehensive review. The work presented in [5] is the continuation of a robust method introduced in many reports [6-9] that employs the concept of a differential sweep equation. While the method is aimed at computing trimming curves in order to remove points that are in the interior of the boundary of the swept volume, the technique by which the authors have formulated the problem is of interest. Surfaces and solids implicitly represented were considered in the sweep equation. We shall adapt this same representation but we will apply the Jacobian rank deficiency method (never before used for implicitly defined equations) to obtain singular sets. These sets will describe (implicitly) surfaces that exist on the exterior and in the interior of the swept volume. Combining these surfaces (referred to as singular surfaces) yields an exact representation of the swept volume.

The method of Jacobian rank deficiency method was developed for parametric surfaces [1-4] and was shown to handle selfintersections, multiple parameter sweeping, and solid property computations. The method was never applied to implicitly defined surfaces because of the lack of formulations for proper representation of such surfaces in a consistent numerical algorithm. This report will outline a systematic method for computing the swept volume of implicit surfaces.

In order to compare with the method recently presented by Blackmore, et al. [5], we shall treat one of the same self- 
intersecting examples addressed therein. We will first address the general problem using the Jacobian rank deficiency method. It will be shown that self-intersections are inherently considered and do not require additional trimming computations.

\section{FORMULATIONS FOR IMPLICIT SWEEP}

In order to remain consistent with the notation used in Blackmore, et al. (1999), where a smooth rigid sweep $\boldsymbol{\sigma}$ may be represented in the form

$\boldsymbol{\sigma}(\mathbf{w})=\boldsymbol{\xi}(t)+\mathbf{A}(t) \mathbf{x}$

where $\mathbf{x}=\left[x_{1}, \ldots, x_{M}\right]^{T}$ and $\mathbf{w}=\left[\mathbf{x}^{T} t\right]^{T}$, and where $\boldsymbol{\xi}$ and $\mathbf{A}$ are, respectively, smooth $\left(=\mathbf{C}^{\infty}\right)$ vector and matrix valued functions with $\boldsymbol{\xi}(0)=\mathbf{0}, \mathbf{A}(0)=\mathbf{I}$ the identity matrix, and $\mathbf{A}(t)$ is a real $(3 \times 3)$ orthonormal matrix (in the case of the $M$ $=3$ ) for every $t \in[0,1]$. For the purpose of developing the formulation, it is required only that $M$ be defined as the set of points satisfying finitely many equalities and inequalities for differentiable functions.

In order to develop a method that is consistent and general for any implicit surface, we will represent the surface $M$ by a number of inequalities, with no restriction on the number of inequalities nor on the type of variables. Let $M$ be represented by

$$
\begin{gathered}
M:=\left\{\mathbf{x}: \ell_{1}^{L} \leq f_{1}(\mathbf{x}) \leq \ell_{1}^{U}, \ldots, \ell_{N}^{L} \leq f_{N}(\mathbf{x}) \leq \ell_{N}^{U},\right. \\
\left.x_{1}^{L} \leq x_{1} \leq x_{1}^{U}, \ldots, x_{k}^{L} \leq x_{k} \leq x_{k}^{U}\right\}
\end{gathered}
$$

where $\mathbf{f}=\left[f_{1}, \ldots, f_{N}\right]^{T}$ and $f_{i}(\mathbf{x})$ denotes an expression representing the surface, $\ell_{i}$ is a limit, the upper subscript $\mathrm{L}$ indicates the lower limit, $\mathrm{U}$ denotes the upper limit, $N$ is the number of expressions, and $k$ is the number of variables. It should be noted that this is the general case, but a surface may be represented by any number of expressions and any number of variables that may or may not have limits.

In order to develop a framework for applying the rank deficiency condition to implicit sweeps, we shall employ a parametrization technique in converting the representation in Eq. (2) to a constraint function. A constraint equation of the form $q_{i}{ }^{L} \leq q_{i} \leq q_{i}{ }^{U}$ is transformed into an equation by introducing a new generalized coordinate $\lambda_{i}$ such that the inequality constraint can be rewritten as

$q_{i}=a_{i}+b_{i} \sin \lambda_{i}$

where $a_{i}=\left(q_{i}{ }^{L}+q_{i}^{U}\right) / 2$ and $b_{i}=\left(q_{i}{ }^{L}-q_{i}^{U}\right) / 2$ are the midpoint and half-range of the inequality constraint [1]. As a result of this transformation, it is now possible to define the sweep by

$$
\boldsymbol{\Phi}(\mathbf{z}):=\left[\begin{array}{c}
\boldsymbol{\sigma}(\mathbf{w}) \\
f_{1}(\mathbf{x})-0.5\left(\ell_{1}^{U}+\ell_{1}^{L}\right)-0.5\left(\ell_{1}^{U}-\ell_{1}^{L}\right) \sin \lambda_{1} \\
\ldots \\
f_{N}(\mathbf{x})-0.5\left(\ell_{N}^{U}+\ell_{N}^{L}\right)-0.5\left(\ell_{N}^{U}-\ell_{N}^{L}\right) \sin \lambda_{N} \\
x_{1}-0.5\left(x_{1}^{U}+x_{1}^{L}\right)-0.5\left(x_{1}^{U}-x_{1}^{L}\right) \sin \lambda_{N+1} \\
\ldots \\
x_{M}-0.5\left(x_{M}^{U}+x_{M}^{L}\right)-0.5\left(x_{M}^{U}-x_{M}^{L}\right) \sin \lambda_{N+M} \\
t-0.5-0.5 \sin \lambda_{N+M+1}
\end{array}\right]
$$

where

$\mathbf{z}=\left[x_{1}, \ldots, x_{N}, t, \lambda_{1}, \ldots, \lambda_{N+M+1}\right]^{T}=\left[z_{1}, \ldots, z_{2 N+M+2}\right]^{T}$ or using partitioned coordinates $\mathbf{z}=\left[\mathbf{w}, \lambda_{1}, \lambda_{2}\right]^{T}$ where $\mathbf{w}=\left[x_{1}, \ldots, x_{N}, t\right]^{T}, \quad \lambda_{1}=\left[\lambda_{1}, \ldots, \lambda_{N}\right]^{T}, \quad$ and $\lambda_{2}=\left[\lambda_{N+1}, \ldots, \lambda_{N+M+1}\right]^{T}$. Note that the parameter $t$ has also been included in $\boldsymbol{\Phi}(\mathbf{z})$.

Rank deficiency conditions first developed by Abdel-Malek and Yeh [1] can now be applied to determine singular sets of the Jacobian $\Phi_{\mathbf{z}}(\mathbf{z})=\partial \Phi / \partial \mathbf{z}$ defined by

$\Phi_{z}=\left[\begin{array}{ccc}\sigma_{w} & 0 & 0 \\ f_{w} & f_{\lambda_{1}} & 0 \\ \mathbf{I} & 0 & \mathbf{w}_{\lambda_{2}}\end{array}\right]$

where $\sigma_{\mathbf{w}}=\partial \sigma / \partial \mathbf{w} ; \mathbf{f}_{\mathbf{w}}=\partial \mathbf{f} / \partial \mathbf{w} ; \mathbf{f}_{\lambda_{1}}=\partial \mathbf{f} / \partial \lambda_{1} ;$ and $\mathbf{w}_{\boldsymbol{\lambda}_{2}}=\partial \mathbf{w} / \partial \boldsymbol{\lambda}_{2}$. The Implicit Function Theorem mandates that there not be open sets in the space of $\mathbf{z}$ in which the constraints are redundant. Redundancy occurs when the Jacobian $\boldsymbol{\Phi}_{\mathbf{z}}$, is rank-deficient which will subsequently define singular surfaces in the manifold. Singularities as a result of the Jacobian rank deficiency condition are denoted by $s_{i}$. An important remark must be made. Compare this Jacobian with that developed for the sweep of parametric surfaces and denoted by

$\Phi_{\mathbf{q}^{*}}=\left[\begin{array}{cc}\sigma_{\mathbf{q}} & \mathbf{0} \\ \mathbf{I} & \mathbf{q}_{\lambda}\end{array}\right]$

where $\mathbf{q}$ is the vector of parameters and $\mathbf{q}^{*}=\left[\mathbf{q}^{T} \boldsymbol{\lambda}^{T}\right]^{T}$. In this case, the singularity of the Jacobian was determined in two distinct categories [4]. However, for the Jacobian of implicit surfaces (Eq. 5), there is an additional submatrix given by $\left[\mathbf{f}_{\mathbf{w}} \mathbf{f}_{\lambda_{1}} \mathbf{0}\right]$. For the Jacobian to have a rank deficiency, a row in this matrix must equal zero, which yields a number of singularities that relate both $\mathbf{f}_{\mathbf{w}}$ and $\mathbf{f}_{\boldsymbol{\lambda}_{1}}$ (typically as a function of several variables). This should become clearer in the illustrative example below. This submatrix generates 
functions that relate the change in the surface coordinates with respect to the sweep parameter.

For a non-square matrix, rank deficiency conditions are imposed by setting the determinants of certain square Jacobian submatrices equal to zero. Their simultaneous solution yields $s_{i}$. These singularities define singular surfaces. The main difference between this formulation and that developed for the sweep of parametric surfaces is the direct generation of singular parametric surfaces (called varieties) in the latter case by direct substitution of $s_{i}$ into $\boldsymbol{\sigma}$.

\section{ILLUSTRATIVE EXAMPLE}

Consider the solid treated in [1] using the SEDE trimming approach. Let $M$ be the solid cylinder in space defined by

$$
M=\left\{\mathbf{x}:-\frac{1}{4} \leq x_{1}^{2}+x_{2}^{2}-\frac{1}{4} \leq 0,-1 \leq x_{3} \leq 1\right\}
$$

The goal is to compute the swept volume of $M$ generated by the sweep

$$
\boldsymbol{\xi}(\mathbf{x}, t)=\left[\begin{array}{c}
0 \\
4 t \\
0
\end{array}\right]+\mathbf{R}(t)\left[\begin{array}{l}
x_{1} \\
x_{2} \\
x_{3}
\end{array}\right]
$$

where $\mathbf{R}$ is a basic rotation matrix about the $\mathbf{x}$-axis defined by

$$
\mathbf{R}(t)=\left[\begin{array}{ccc}
1 & 0 & 0 \\
0 & \cos (\pi t) & -\sin (\pi t) \\
0 & \sin (\pi t) & \cos (\pi t)
\end{array}\right]
$$

and where the sweep parameter $t$ is bounded as $0 \leq t \leq 1$. The sweep can be written as

$$
\begin{gathered}
\boldsymbol{\xi}(\mathbf{z})=\left[\begin{array}{c}
x_{1} \\
4 t+x_{2} \cos (\pi t)-x_{3} \sin (\pi t) \\
x_{2} \sin (\pi t)+x_{3} \cos (\pi t) \\
x_{1}^{2}+x_{2}^{2}-0.25-0.25 \sin \left(\lambda_{1}\right) \\
x_{3}-\sin \left(\lambda_{2}\right) \\
t-0.5-0.5 \sin \left(\lambda_{3}\right) \\
z_{1}
\end{array}\right] \\
=\left[\begin{array}{c}
z_{2} \sin \left(\pi z_{4}\right)+z_{3} \cos \left(\pi z_{4}\right) \\
z_{1}^{2}+z_{2}^{2}-0.25-0.25 \sin \left(z_{5}\right) \\
z_{3}-\sin \left(z_{6}\right) \\
z_{4}-0.5-0.5 \sin \left(z_{7}\right)
\end{array}\right]
\end{gathered}
$$

where $\mathbf{z}=\left[z_{1}, z_{2}, z_{3}, z_{4}, z_{5}, z_{6}, z_{7}\right]^{T}$.

To apply the rank deficiency condition, and because the Jacobian is non-square (dimension $6 \times 7$ ), we compute the seven $(6 \times 6)$ sub-Jacobians (denoted by $\mathrm{J}_{\mathrm{i}}$ ), compute their determinants, and equate them to zero. The simultaneous zeros of these determinants are singular sets that yield the socalled Singular Surfaces. The determinants are evaluated as: $\operatorname{det}\left[\mathrm{J}_{1}\right]=0$

$\operatorname{det}\left[\mathrm{J}_{2}\right]=$

$0.25 \cos \left(\pi z_{4}\right) \cos z_{5} \cos z_{6} \cos z_{7}-0.19635 z_{3} \cos ^{2}\left(\pi z_{4}\right) \cos z_{5} \cos z_{6} \cos z_{7}$

$$
-0.19635 z_{3} \sin ^{2}\left(\pi z_{4}\right) \cos z_{5} \cos z_{6} \cos z_{7}=0
$$

which can be further simplified to $\operatorname{det}\left[\mathrm{J}_{2}\right]=\cos z_{5} \cos z_{6} \cos z_{7}$ $\left(-0.19635 z_{3}+0.25 \cos \left(\pi z_{4}\right)\right)=0$

Note that derivations and algebraic manipulations of the determinants of the Jacobian are carried out using a symbolic manipulator (Mathematica $\left.{ }^{\circledR}\right)$.

$\operatorname{det}\left[\mathrm{J}_{3}\right]=\cos z_{5} \cos z_{6} \cos z_{7}\left(-0.196 z_{2}+0.25 \sin \left(\pi z_{4}\right)\right)=0$

$\operatorname{det}\left[\mathrm{J}_{4}\right]=-0.0625 \cos z_{5} \cos z_{6} \cos z_{7}=0$

$\operatorname{det}\left[\mathrm{J}_{5}\right]=\cos z_{6} \cos z_{7}\left(\pi z_{2} z_{3}-4 z_{2} \cos \left(\pi z_{4}\right)\right)=0$

$\operatorname{det}\left[\mathrm{J}_{6}\right]=\cos z_{5} \cos z_{7}\left(0.196 z_{2}-0.25 \sin \left(\pi z_{4}\right)\right)=0$

$\operatorname{det}\left[\mathrm{J}_{7}\right]=0.125 \cos z_{5} \cos z_{6}=0$

The singularity sets as a result of the simultaneous solution of Eqs. (11-17) are:

$\mathrm{s}_{1}=\left\{z_{5}=\pi / 2\right.$, and $\left.z_{6}=\pi / 2\right\}, \mathrm{s}_{2}=\left\{z_{5}=\pi / 2\right.$, and $\left.z_{6}=-\pi / 2\right\}$, $\mathrm{s}_{3}=\left\{z_{5}=-\pi / 2\right.$, and $\left.z_{6}=\pi / 2\right\}, \mathrm{s}_{4}=\left\{z_{5}=-\pi / 2\right.$, and $\left.z_{6}=-\pi / 2\right\}$, $\mathrm{s}_{5}=\left\{z_{5}=\pi / 2\right.$, and $\left.z_{7}=\pi / 2\right\}, \mathrm{s}_{6}=\left\{z_{5}=\pi / 2\right.$, and $\left.z_{7}=-\pi / 2\right\}$, $\mathrm{s}_{7}=\left\{z_{5}=-\pi / 2\right.$, and $\left.z_{7}=\pi / 2\right\}, \mathrm{s}_{8}=\left\{z_{5}=-\pi / 2\right.$, and $\left.z_{7}=-\pi / 2\right\}$, $\mathrm{s}_{9}=\left\{z_{5}=\pi / 2\right.$, and $\left.z_{2}=0\right\}, \mathrm{s}_{10}=\left\{z_{5}=-\pi / 2\right.$, and $\left.z_{2}=0\right\}$,

$\mathrm{s}_{11}=\left\{z_{6}=\pi / 2\right.$, and $\left.z_{7}=\pi / 2\right\}, \mathrm{s}_{12}=\left\{z_{6}=\pi / 2\right.$, and $\left.z_{7}=-\pi / 2\right\}$, $\mathrm{s}_{13}=\left\{z_{6}=-\pi / 2\right.$, and $\left.z_{7}=\pi / 2\right\}, \mathrm{s}_{14}=\left\{z_{6}=-\pi / 2\right.$, and $\left.z_{7}=-\pi / 2\right\}$, $\mathrm{s}_{15}=\left\{z_{5}=\pi / 2\right.$, and $\left.z_{3}=4 / \pi \cos \left(\pi z_{4}\right)\right\}, \quad \mathrm{s}_{16}=\left\{z_{5}=-\pi / 2\right.$, and $z_{3}=4 / \pi$ $\left.\cos \left(\pi z_{4}\right)\right\}, \quad \mathrm{s}_{17}=\left\{z_{6}=\pi / 2\right.$, and $\left.z_{2}=1.27 \sin \left(\pi z_{4}\right)\right\}, \mathrm{s}_{18}=\left\{z_{6}=-\pi / 2\right.$, and $\left.z_{2}=0.25 / 0.196 \sin \left(\pi z_{4}\right)\right\}$;

For each singular set, a geometric entity must be determined. Depending on the rank of the Jacobian deficiency, this entity could be in the form of a curve or a surface. To illustrate, consider $s_{1}=\left\{z_{5}=\pi / 2\right.$, and $\left.z_{6}=\pi / 2\right\}$. For $z_{5}=\pi / 2$ (recall that $\left.z_{5}=\lambda_{1}\right)$ the parametrization $x_{1}^{2}+x_{2}^{2}=0.25+0.25 \sin \left(\lambda_{1}\right)$, yields $x_{1}^{2}+x_{2}^{2}=0.25$. Similarly, $z_{6}=\pi / 2$ (recall that $z_{6}=\lambda_{2}$ ) indicates that $x_{3}=1$ ). Hence this singularity is defined by $\mathrm{s}_{1}=\left\{x_{1}^{2}+x_{2}^{2}=0.25, x_{3}=1\right\}$. For each singularity set, a similar analysis is performed and all singularities are listed below.

$$
\begin{aligned}
& \mathrm{s}_{2}=\left\{x_{1}^{2}+x_{2}^{2}=0.25, x_{3}=-1\right\} . \\
& \mathrm{s}_{3}=\left\{x_{1}^{2}+x_{2}^{2}=0, x_{3}=1\right\}, \mathrm{s}_{4}=\left\{x_{1}^{2}+x_{2}^{2}=0, x_{3}=-1\right\}, \\
& \mathrm{s}_{5}=\left\{x_{1}^{2}+x_{2}^{2}=0.25, t=1\right\}, \mathrm{s}_{6}=\left\{x_{1}^{2}+x_{2}^{2}=0.25, t=0\right\}, \\
& \mathrm{s}_{7}=\left\{x_{1}^{2}+x_{2}^{2}=0, t=1\right\}, \mathrm{s}_{8}=\left\{x_{1}^{2}+x_{2}^{2}=0, t=0\right\}, \\
& \mathrm{s}_{9}=\left\{x_{1}^{2}+x_{2}^{2}=0.25, x_{2}=0\right\}, \mathrm{s}_{10}=\left\{x_{1}^{2}+x_{2}^{2}=0, x_{2}=0\right\},
\end{aligned}
$$


$\mathrm{s}_{11}=\left\{x_{3}=1, t=1\right\}, \mathrm{s}_{12}=\left\{x_{3}=1, t=0\right\}$,

$\mathrm{s}_{13}=\left\{x_{3}=-1, t=1\right\}, \mathrm{s}_{14}=\left\{x_{3}=-1, t=0\right\}$;

$\mathrm{s}_{15}=\left\{x_{1}^{2}+x_{2}^{2}=0.25, x_{3}=4 / \pi \cos (\pi t)\right\}, \mathrm{s}_{16}=\left\{x_{1}^{2}+x_{2}^{2}=0\right.$, $\left.x_{3}=4 / \pi \cos (\pi t)\right\}$,

$\mathrm{s}_{17}=\left\{x_{3}=1, x_{2}=0.25 / 0.196 \sin (\pi t)\right\}, \mathrm{s}_{18}=\left\{x_{3}=-1, x_{3}=0.25 / 0.196\right.$ $\sin (\pi t)\}$

Note that singular sets $\mathrm{s}_{15}-\mathrm{S}_{18}$ have variables that are parametrized as a function of another variable. Also note that sets $s_{3}, s_{4}, s_{7}, s_{8}, s_{10}, s_{16}, s_{17}, s_{18}$ are curves. Surfaces due to singularity sets $\mathrm{s}_{1}, \mathrm{~s}_{2}, \mathrm{~s}_{5}, \mathrm{~s}_{6}, \mathrm{~s}_{9}, \mathrm{~s}_{11}, \mathrm{~s}_{12}, \mathrm{~s}_{13}, \mathrm{~s}_{14}, \mathrm{~s}_{15}$, are depicted in Fig. 1.
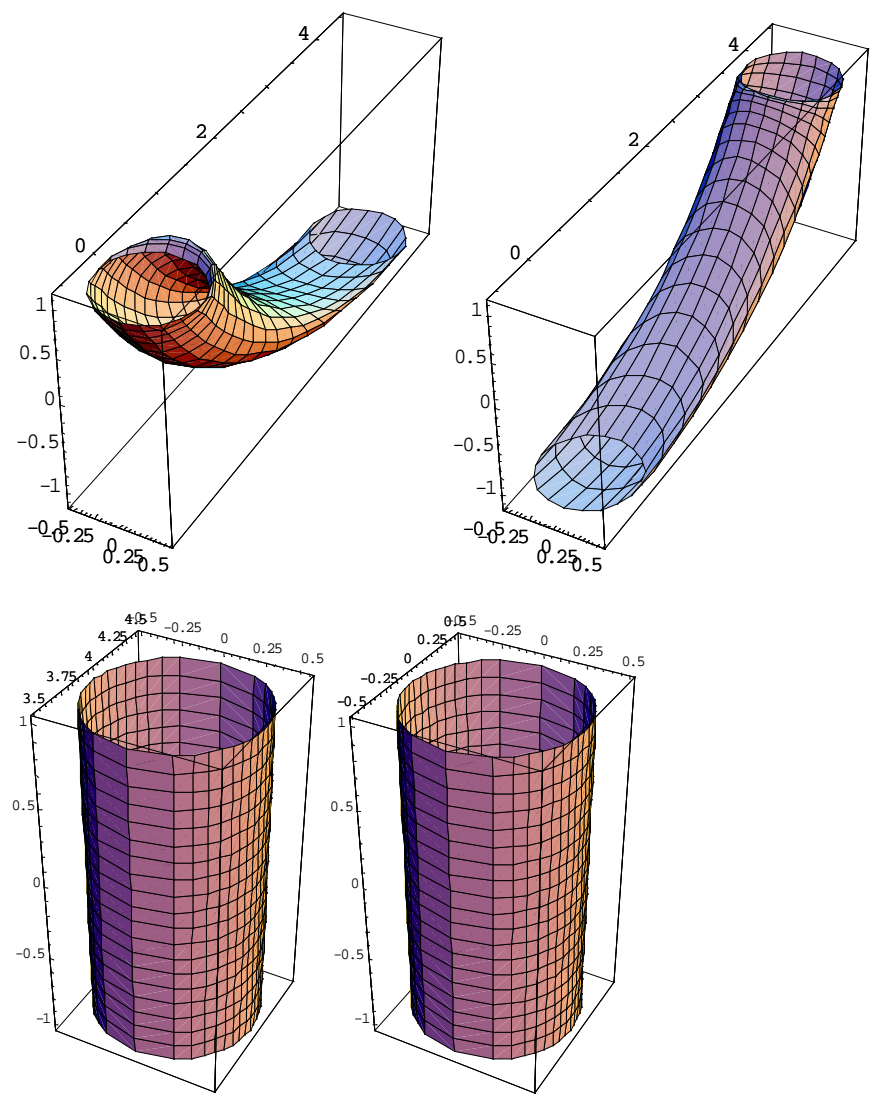

Fig. 1 Surfaces due to (a) singularity $s_{1}$ (b) singularity $s_{2}$ (c) singularity $\mathrm{s}_{5}$ and (d) singularity $\mathrm{s}_{6}$
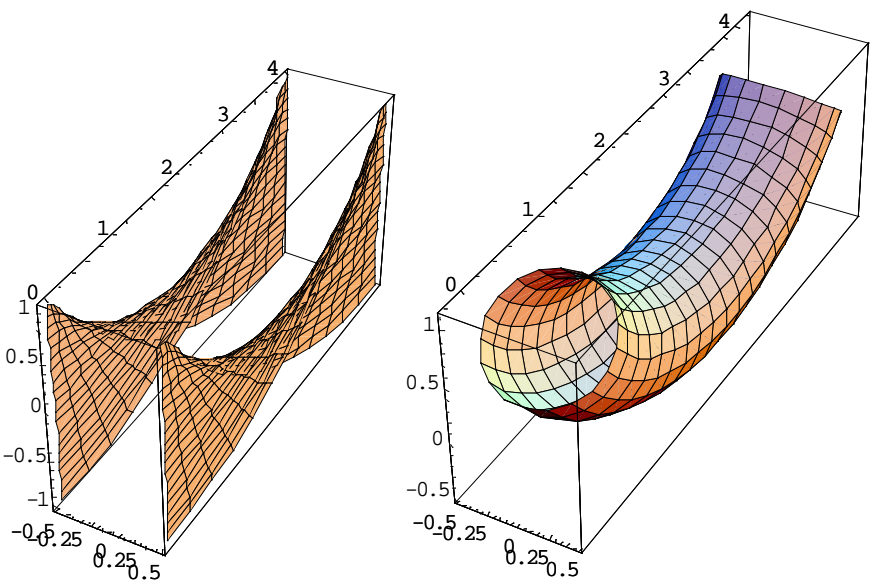

Fig. 1 (e) singularity $s_{9}$ and (f) singularity $s_{15}$
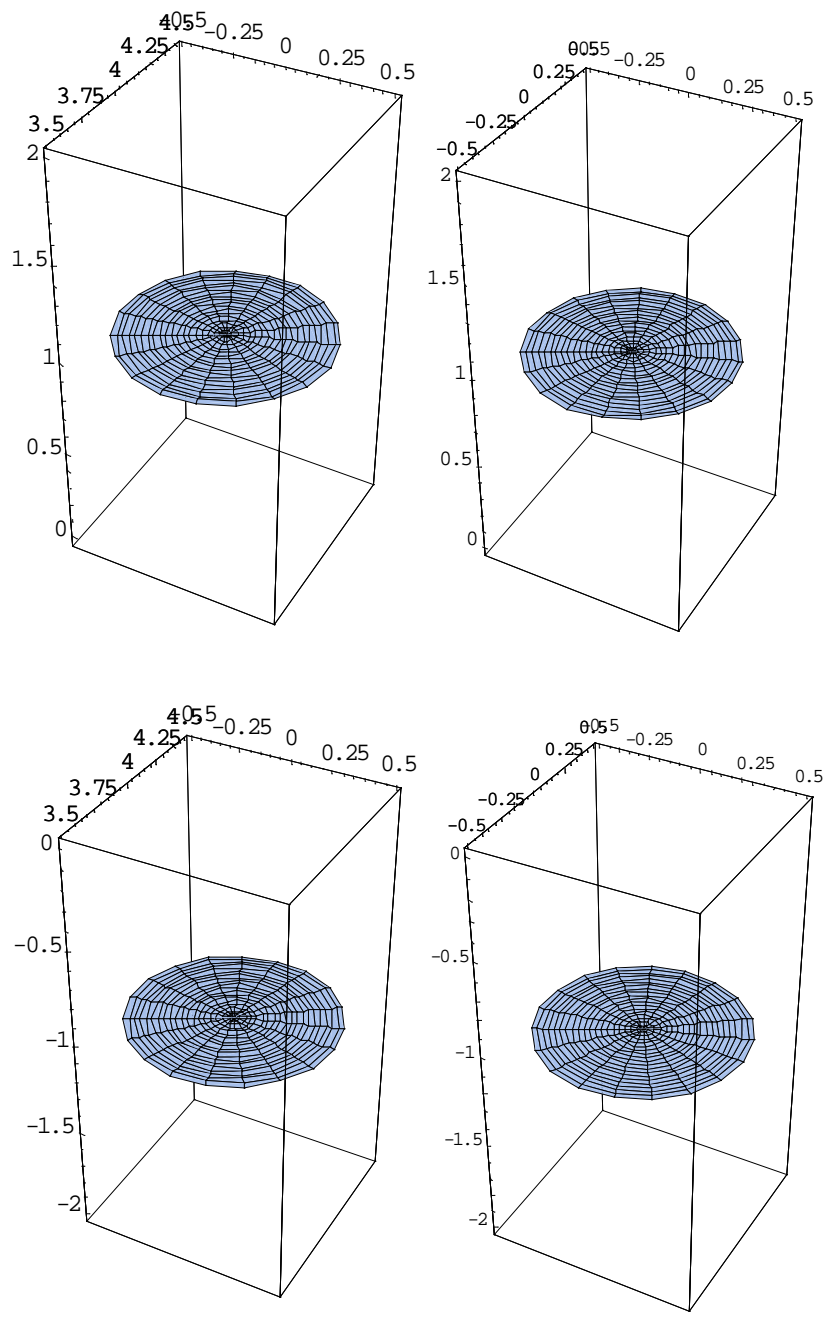

Fig. 1 (h) singularity $s_{11}(i)$ singularity $s_{12}$ and (j) singularity $\mathrm{s}_{13}(\mathrm{k})$ singularity $\mathrm{s}_{14}$ 
Combining all surfaces yields the swept volume as shown in Fig. 2.

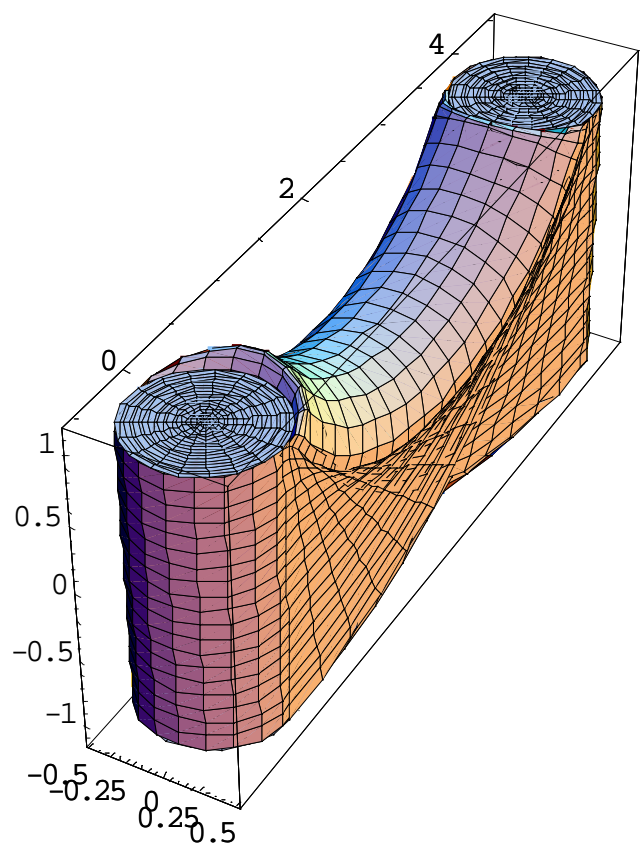

Fig. 2 The swept volume (same example treated in Blackmore, et al. using SEDE trimming methods)

\section{PARAMETRIC SWEEP}

In order to compare between the two formulations for the Jacobian rank deficiency condition (for both implicit and parametric surfaces), we shall examine the same sweep but for the object we use a parametric representation of the solid cylinder given by

$$
\mathbf{x}\left(q_{1}, q_{2}, q_{4}\right)=\left[\begin{array}{lll}
q_{4} \cos q_{1} & q_{4} \sin q_{1} & q_{2}
\end{array}\right]^{T}
$$

on the surface parametrized as $\boldsymbol{\xi}\left(q_{3}\right)=\left[\begin{array}{lll}0 & 4 q_{3} & 0\end{array}\right]^{T}$ with the same rotation matrix as above $\mathbf{R}\left(q_{3}\right)$,

where $\quad-\pi \leq q_{1} \leq \pi, \quad-1 \leq q_{2} \leq 1, \quad 0 \leq q_{3} \leq 1, \quad$ and $0 \leq q_{4} \leq 0.5$. The sweep equation is defined by

$$
\sigma(\mathbf{q})=\left[\begin{array}{c}
q_{4} \cos q_{1} \\
4 q_{3}+q_{4} \cos \left(\pi q_{3}\right) \sin q_{1}-q_{2} \sin \left(\pi q_{3}\right) \\
q_{2} \cos \left(\pi q_{3}\right)+q_{4} \sin q_{1} \sin \left(\pi q_{3}\right)
\end{array}\right]
$$

The Jacobian is a $3 \times 4$ non-square matrix. Applying the rank deficiency conditions yields four sub-Jacobians that are equated to zero as

$$
\begin{aligned}
& \operatorname{det}\left[J_{1}\right]=q_{4}\left(-\pi q_{2}+4 \cos \left(\pi q_{3}\right)\right) \sin q_{1}=0 \\
& \operatorname{det}\left[J_{2}\right]=q_{4}=0 \\
& \operatorname{det}\left[J_{3}\right]=q_{4}\left(\pi q_{4} \sin q_{1}-4 \sin \left(\pi q_{3}\right)\right)=0 \\
& \operatorname{det}\left[J_{4}\right]=\cos q_{1}\left(\pi q_{2}-4 \cos \left(\pi q_{3}\right)\right)=0
\end{aligned}
$$

Simultaneous solutions to the above four equations yields the singular sets listed below. Considering the definition for rank deficency, reduced rank deficency, and constraint singularity [1], the total sets are computed as follows:

$p_{1}=\left\{q_{1}=-\pi, q_{4}=0.5\right\}, \quad p_{2}=\left\{q_{1}=0, q_{4}=0.5\right\}, \quad p_{3}=\left\{q_{2}=-1\right.$, $\left.q_{3}=0\right\}, p_{4}=\left\{q_{2}=-1, q_{3}=1\right\}$,

$p_{5}=\left\{q_{2}=1, q_{3}=0\right\}, p_{6}=\left\{q_{2}=1, q_{3}=1\right\}, \quad p_{7}=\left\{q_{2}=-1, q_{4}=0.5\right\}$, $p_{8}=\left\{q_{2}=1, q_{4}=0.5\right\}$,

$p_{9}=\left\{q_{3}=0, q_{4}=0.5\right\}, \quad p_{10}=\left\{q_{3}=1, q_{4}=0.5\right\}, \quad p_{11}=\left\{q_{3}=0.318\right.$ $\left.\operatorname{ArcCos}\left(0.785 q_{2}\right), q_{4}=0.5\right\}$

These singular sets are equivalent (in parametric form) to those computed using the implicit sweep formulation. In particular, consider the singularity $p_{11}$ (in the parametric formulation ) where $q_{3}=0.318 \operatorname{ArcCos}\left(0.785 q_{2}\right)$. This is equivalent to $x_{3}=4 / \pi \cos (\pi t)$ (or $t=(1 / \pi) \operatorname{Arc} \operatorname{Cos}\left(x_{3} \pi / 4\right)$ $\left.=.318 \operatorname{Arc} \operatorname{Cos}\left(.785 x_{3}\right)\right)$ in $s_{15}$ and $s_{16}$.

\section{EXAMPLE 2}

Consider the sweep of the surface given by

$$
M=\left\{\mathbf{x}: x_{3}=x_{1}^{2}-x_{2}^{2},-2 \leq x_{1} \leq 2,-2 \leq x_{2} \leq 2\right\}
$$

with the sweep given by $\boldsymbol{\xi}(t)=\left[\begin{array}{lll}0 & t & t\end{array}\right]^{T}, ; 0 \leq t \leq 4$, $\mathbf{A}=\mathbf{I}$ (identity) and $\mathbf{x}=\left[\begin{array}{lll}x_{1} & x_{2} & x_{3}\end{array}\right]^{T}$. Using the Jacobian rank deficiency conditions, there are seven singular sets that yield the following surfaces

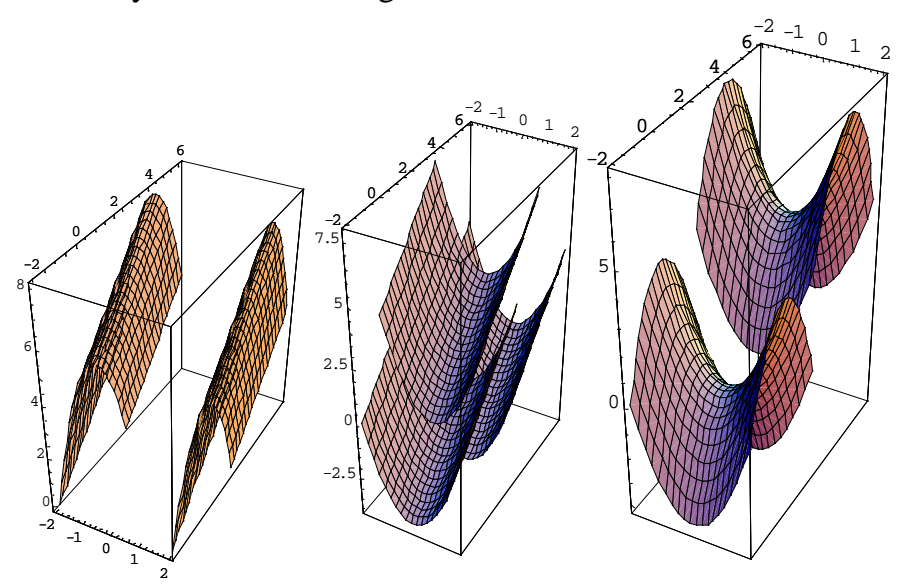

Fig. 3 Singular surfaces of Example 2

The complete swept volume is shown in Fig. 4. 


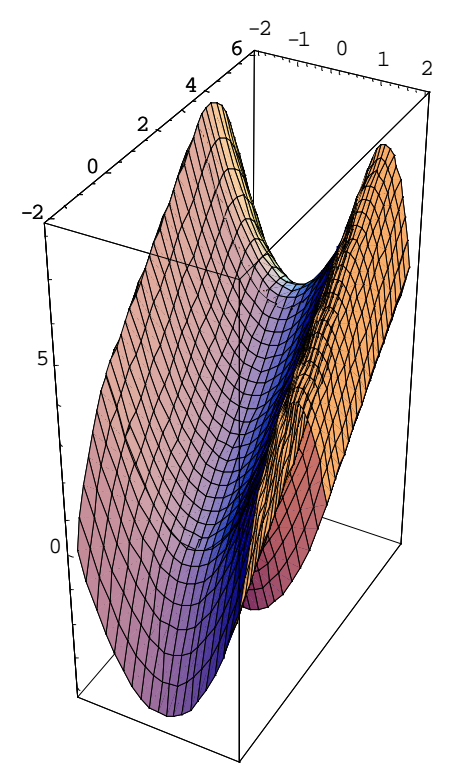

Fig. 4 Swept volume

\section{CONCLUSIONS}

The method based on a Jacobian rank deficiency condition developed for computing the sweep of parametric surfaces was expanded in this report to a broadly applicable formulation for implicit surfaces. This was accomplished by applying the parametrization method of an implicit surface.

Note that in the foregoing analysis, neither the dimension of $\mathbf{x}$ nor the number of inequality expressions has been limited. As a result, the formulation is valid for $n$-dimensional manifolds.

Also note that the resulting swept volume is exact as no approximations have been made. It is also noted that the formulation is developed as an algorithm suitable for computer implementation using commercial symbolic manipulators with an ability to perform derivation and plotting of surfaces.

\section{REFERENCES}

1. Abdel-Malek, $\mathrm{K}$ and Yeh, HJ "Geometric Representation of the Swept Volume Using Jacobian Rank Deficiency Conditions," Computer-Aided Design, Vol. 29, No. 6, (1997), pp457-468.

2. Abdel-Malek, K, Yeh, HJ, and Othman, S, 1998, " Swept Volumes: Void and Boundary Identification," ComputerAided Design, Vol. 30, No. 13, pp1009-1018.

3. Abdel-Malek, K, Yeh, HJ, and Rouwady, R, 1997b "A Unified Formulation for Swept Volume and Robot Workspace Analysis," Proceedings of the ASME Design Automation Conference, Sacramento, CA.

4. Abdel-Malek, K, Seaman, W, and Yeh, HJ, "NC Verification of up to 5 Axis Machining Processes using Manifold Stratification," (in press) ASME Journal of Manufacturing Science and Engineering.
5. Abdel-Malek, K. and Othman, S., (1999), "Multiple sweeping using the Denavit-Hartenber representation method", Computer-Aided Design, Vol. 31, pp. 567-583.

6. Blackmore, D, Samulyak, R, and Leu, MC, "Trimming swept volumes", Computer-Aided Design, 31(3), 215-224.

7. Blackmore, D, Leu, MC, Wang, LP, Jiang, H, 1997, "Swept Volumes: a retrospective and prospective view," Neural Parallel and Scientific Computations, 5, pp81-102.

8. Blackmore, D and Leu, M C, 1992, 'Analysis of Swept Volume via Lie Groups and Differential Equations' Int Journal of Robotics Research Vol. 11(6), pp516-537.

9. Leu, M C, Park, S H, and Wang, K K 'Geometric Representation of Translational Swept Volumes and Its Applications' ASME Journal of Engineering for Industry, Vol. 108 (1986) pp113-119.

10. Blackmore, D, Leu, M C, and Shih, F 'Analysis and Modeling of Deformed Swept Volumes' Computer-Aided Design Vol. 25(4) (1994), pp315-326. 